\title{
$c$ axes from radar depolarization experiments at Upstream B Camp, Antarctica, in 1991-92
}

\author{
C. LiU, C. R. Bentley and N. E. Lord \\ Geophysical and Polar Research Center, University of Wisconsin-Madison, Madison, WI 53706, U.S.A.
}

\begin{abstract}
Thirty-nine $50 \mathrm{MHz}$ radar polarization experiments were performed in 1991-92 near Lpstream B Camp, Antarctica, along two lines perpendicular to flow, 1.4 and $2 \mathrm{~km}$ long and $900 \mathrm{~m}$ apart. For each of the experiments, which were at $100 \mathrm{~m}$ intervals, the receiving antenna was held fixed, alternately parallel and perpendicular to flow, while the transmitting antenna was rotated in $15^{\circ}$ increments through a full circle twice, once for cach oricntation of the recciving antenna. The data consist of echo-amplitude measurements from the bottom of the ice. Assuming a model of the ice sheet as a crystalline medium with axial symmetry, the azimuths of the symmetry axis and the cosines of the phase shifts between extraordinary and ordinary waves can be estimated from the variations in amplitude with orientation of the transmitting antenna. The results from bottom cchocs show an abrupt change in the axis of symmetry in a distance of only $100 \mathrm{~m}$. This suggests that the experimental lines cross the boundary between two blocks of ice with different stress histories.
\end{abstract}

\section{INTRODUCTION}

Crystalline fabric is one of the most important factors in the flow law for ice, so it is important to find ways of measuring it. In this paper we discuss the application of a radar depolarization method for measuring the $c$-axis orientation at Upstream B Camp, Antarctica.

Scveral workers have found, by observing the dependence of the strength of radio signals reflected from the base of the ice on the orientations of the transmitting and receiving antennas, that large ice sheets can change the polarization of radio waves transmitted through them Jiracek, 1967; Bentley, 1975; Hargreaves, 1977; Woodruff and Doake, 1979; Doake, 1981). Usually, an elliptically polarized reflected wave is received if a linearly polarized wave is transmitted. Occasionally, the maximum echo strength appears when the transmitting and receiving antennas are perpendicular to cach other and sometimes the received echo strength is independent of the relative orientation of the antennas. All of these phenomena can be cxplained if the ice sheet behaves as a birefringent medium because of anisotropy in the permittivity of ice. This anisotropy is too weak to have been detected in the laboratory until recently it has now been observed at $9.7 \mathrm{GHz}$ by Fujita and others (1993), who also cited reasons for belicving the same permittivities should also obtain at the frequency of sounding radars.

Several other factors could cause a change in the polarization state of a radio wave in a polar ice sheet (Hargreaves, 1977). (1) In the polar regions the geomagnetic field has a large vertical component; Faraday rotation might then create a small rotation of the polarization plane, which would add rather than cancel on the return path. (2) Air bubbles distributcd anisotropically in the ice could scatter waves unequally depending on their polarization. (3) The distribution of crevasses in the ice might affect the polarization state. (4) The attenuation during passage through the ice or the reflection coefficient at a surface might depend on the polarization of the transmitted or incident wave.

We discount the first threc factors for the following reasons. (1) The geomagnetic field effect is irrelevant to changes in orientation as it would be constant over the small area of the experiment. (2) As shown by Hargreaves (1978), the effect of air bubbles would be too small. (3) Owing to their wide spacing, buried crevasses could only affect a small fraction of our individual experiments at most. That leaves anisotropic attenuation and reflection still to be considered in addition to anisotropy in the permittivity.

We first assume that the ice sheet behaves as an idcalized single crystal whose axis of symmetry we call the "effective $c$ axis". When a single incident plane wave propagates through a birefringent medium, such as a. single crystal of icc, the wave divides into "ordinary" and "extraordinary" waves, traveling with different wave speeds, whose electrical vectors oscillate perpendicular to each other. These two waves can be observed arriving at different times and with different polarizations after propagation through the medium; the time difference, together with the wave speed and wavelength, gives a measure of the phase shift. We estimate the azimuth of the cffcctivc $c$ axis and the cosine of the phase difference between the bottom-reflected ordinary and extraordinary waves at each experimental site from the dependence of the amplitudes of the reflections on the orientations of the transmitting and receiving antennas. From the cosine of the phase difference, we estimate the angle between the 
effective $c$ axis and the direction of wave propagation. Finally, we discuss how a particular distribution of $c$ axes would affect the results.

The data were collected during the 1991-92 austral summer at UpB Camp, Antarctica. Thirty-nine $50 \mathrm{MHz}$ radar depolarization experiments were performed close to the " 34 " and " 35 " lines and between the " $A$ " and " $C$ " lines of The Ohio State Lniversity strain-measurement grid where I.M. Whillans (personal communication, 1991) reported strong shear strain (Fig. l; for a map of the whole strain grid see Clarke and Bentley (1994)). The upstream profile ( $\mathrm{P}$ line) was $2 \mathrm{~km}$ long and the downstream one ( $(2$ line), located $900 \mathrm{~m}$ away, was $1.4 \mathrm{~km}$ long. Both profiles ran perpendicular to the direction of ice-stream movement. The individual experiments were locatcd $100 \mathrm{~m}$ apart on each profile. For each experiment, the receiving antenna, which was a half-wave dipole, was placed on the profile line. The transmitting antenna, an identical half-wave dipole, was positioned $20 \mathrm{~m}$ downstream from the receiving antenna. During sounding the transmitting antenna was twice rotated in $15^{\circ}$ increments through a full circle, once each with the receiving antenna parallel to flow and perpendicular to flow. The data, comprising echo-amplitude measurements from the bottom of the ice and from internal layers, were recorded digitally on magnetic tapes. Three additional experiments (at P5, P7 and P9) were done with receiving and transmitting antennas separatcd along the profile; no difference was detected relative to the experiments with antennas separated along flow.

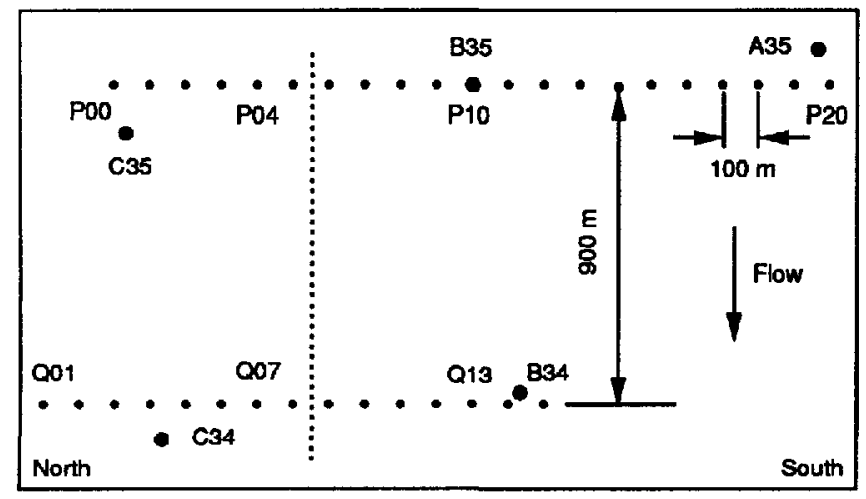

Fig. 1. The locations of depolarization experiments on Ice Stream $B$. The larger dots and numbers denote poles of The Ohio Stale University strain grid; smaller dots and numbers refer to our profiles. The arrow marked "Flow" shows the direction of ice movement. The dotted line indicates the boundary between sections of the profiles as demarcated in Figures 4 and 5.

\section{THEORETICAL MODEL}

\section{Uniaxial model}

We assume that ice behaves as a uniaxial birefringent medium and consider a radio wave propagating at normal incidence from the icc surface with electric-field amplitude $E_{\mathrm{t}}$. Upon passage through the ice, the wave separates into extraordinary and ordinary waves, which, after reflection at depth $h$, can be expressed by their electric fields (Figure 2 shows the geometry)

$$
\begin{aligned}
& E_{\mathrm{e}}=K_{\mathrm{e}} E_{\mathrm{t}} \cos (\alpha-\theta) \exp \left(i \omega\left(t-2 h / V_{\mathrm{e}}\right)\right) \\
& E_{\mathrm{o}}=K_{\mathrm{o}} E_{\mathrm{t}} \sin (\alpha-\theta) \exp \left(i \omega\left(t-2 h / V_{0}\right)\right),
\end{aligned}
$$

where $\omega$ is the angular frequency, $\alpha$ is the angle between the transmitting antenna and the flow direction, $\theta$ is the angle in the horizontal plane between the $c$-axis orientation and flow, $V_{\mathrm{e}}$ is the extraordinary-wave speed, $V_{o}$ is the ordinary-wave speed, $K_{\mathrm{e}}$ and $K_{o}$ are the overall attenuation coefficients of the extraordinary and ordinary waves, respectively, due to absorption, reflection loss and geometrical attenuation, and $t$ is the two-way travel time.

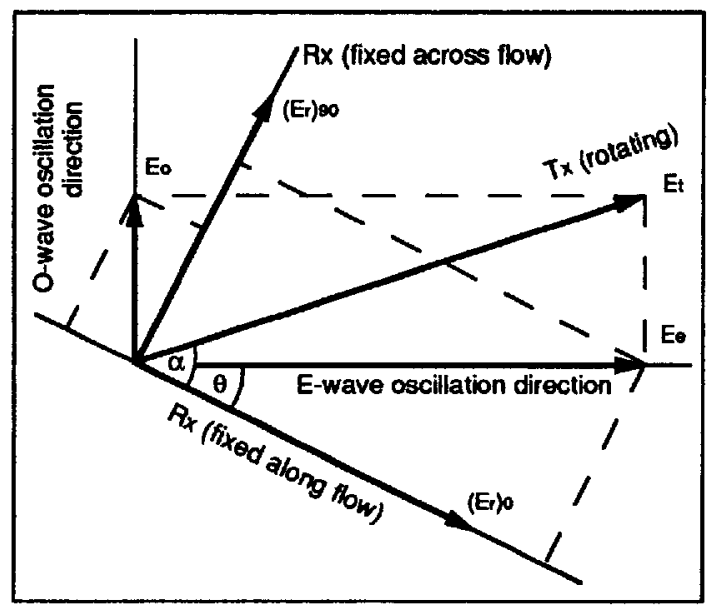

Fig. 2. Sketch of a polarization experiment. The (horizontal) snowe surface lies in the plane of the diagram and the wave propagates vertically, $R x$ and $T_{x}$ denole receizing and transmitting antennas, respectively. The two $R x$ directions are altemative, not simultaneous, orientalions.

The relative powers received along and across flow, $\left(E_{\mathrm{r}}\right)_{0}$ and $\left(E_{\mathrm{r}}\right)_{90}$, respectively, are then (Doake, 1981)

$$
\begin{aligned}
\left(\frac{\left|\left(E_{\mathrm{r}}\right)_{0}\right|}{K_{0} E_{\mathrm{l}}}\right)^{2}= & r^{2} \cos ^{2} \theta \cos ^{2}(\alpha-\theta)+\sin ^{2} \theta \sin ^{2}(\alpha-\theta) \\
& -\frac{1}{2} r \sin 2 \theta \sin 2(\alpha-\theta) \cos \phi
\end{aligned}
$$

and

$$
\begin{aligned}
\left(\frac{\left|\left(E_{\mathrm{r}}\right)_{90}\right|}{K_{0} E_{\mathrm{t}}}\right)^{2}= & r^{2} \sin ^{2} \theta \cos ^{2}(\alpha-\theta)+\cos ^{2} \theta \sin ^{2}(\alpha-\theta) \\
& +\frac{1}{2} r \sin 2 \theta \sin 2(\alpha-\theta) \cos \phi
\end{aligned}
$$

where

$$
\phi=2 \omega h\left(\frac{1}{V_{\theta}}-\frac{1}{V_{0}}\right)
$$

is the phase difference between extraordinary and ordinary waves, and $r=K_{\mathrm{e}} / K_{0}$. The unknowns of interest are $\theta$ and $\cos \phi$; they can be found by least squares from the 24 sets of $\alpha$ and $\left(E_{\mathrm{r}}\right)_{0}$ (or $\left.\left(E_{\mathrm{r}}\right)_{90}\right)$ that have been measured in the ficld. For an isotropic reflector $r=1$, and, from Equations (1) and (2) 


$$
\begin{aligned}
& 2\left(\frac{\left|\left(E_{\mathrm{r}}\right)_{0}\right|}{K_{\mathrm{o}} E_{\mathrm{t}}}\right)^{2}=1+X \cos 2 \alpha+Y \sin 2 \alpha \\
& 2\left(\frac{\left|\left(E_{\mathrm{r}}\right)_{90}\right|}{K_{\mathrm{o}} E_{\mathrm{t}}}\right)^{2}=1-X \cos 2 \alpha-Y \sin 2 \alpha
\end{aligned}
$$

where $X=\cos ^{2} 2 \theta+\sin ^{2} 2 \theta \cos \phi$ and $Y=\cos 2 \theta \sin 2 \theta$ $(1-\cos \phi)$, whence

$$
\tan 2 \theta=\frac{1-X}{Y}
$$

and

$$
\cos \phi=1-\frac{Y^{2}+(1-X)^{2}}{1-X}
$$

From Equations (4) and $(5),\left(K_{\mathrm{o}} E_{\mathrm{t}}\right)^{2}$ can be found using the conservation of power:

$$
\left(\frac{\left|\left(E_{\mathrm{r}}\right)_{90}\right|}{K_{\mathrm{o}} E_{\mathrm{t}}}\right)^{2}+\left(\frac{\left|\left(E_{\mathrm{r}}\right)_{0}\right|}{K_{\mathrm{o}} E_{\mathrm{t}}}\right)^{2}=1
$$

$X$ and $Y$ can be found by least squares from Equations (4) and (5), then $\tan 2 \theta$ and $\cos \phi$ follow directly from Equations (6) and (7). Neither $\theta$ nor $\phi$ is a single-valued function of $X$ and $Y$. For any solution, $\theta_{1}$, of Equation (6), $\theta_{1}+\pi / 2$ is also a solution. That means that a rotation of the $r$-axial pattern through $90^{\circ}$ in the horizontal plane would not affect the results. Similarly, for any solution $\phi_{1}$ of Equation (7), $\phi_{1}+2 n \pi$ and $(2 n-1) \pi-\phi_{1}$ are also solutions for any integer $n-$ that simply reflects the indeterminacy of the total phase shift between the ordinary and extraordinary waves. Note, however, that $\tan 2 \theta$ and $\cos \phi$ can be found whatever the wave speeds (so long as they are different).

In the vertical cross-section containing the $c$ axis, called the principal plane, we can diagram the velocity functions (wave speeds as functions of the angle between the $c$ axis and the vertical) (Fig. 3). The ordinary wave, with polarization normal to the principal plane, propagates with a speed that is independent of direction, i.e. its velocity function is circular. The velocity function for the extraordinary wave, which oscillates in the principal plane, is an ellipsc.

According to Huygen's principle, the extraordinary wave that has a horizontal wave front actually propagates at a slight angle, $\xi$, to the vertical (unless the $c$ axis is horizontal or vertical). From the geometry in Figure 3, the angle between ordinary and extraordinary rays can be shown to be

$$
\xi=\tan ^{-1}\left[\frac{\left(n_{\mathrm{e}}{ }^{2}-n_{\mathrm{o}}{ }^{2}\right) \tan \beta}{\left(n_{\mathrm{e}}{ }^{2}+n_{\mathrm{o}}{ }^{2}\right) \tan \beta}\right]
$$

where $n_{0}=C / V_{0}$ and $n_{\mathrm{e}}=C / V_{\mathrm{e}}^{\prime}$ are the refractive indexes of the ordinary and extraordinary waves, respectively, $V_{\mathrm{B}}^{\prime}$ is the minimum of the extraordinary- wave speed for propagation perpendicular to the $c$ axis), $C$ is the specd of light and $\beta$ is the angle between the vertical and the $c$ axis. The permittivity difference between extraordinary

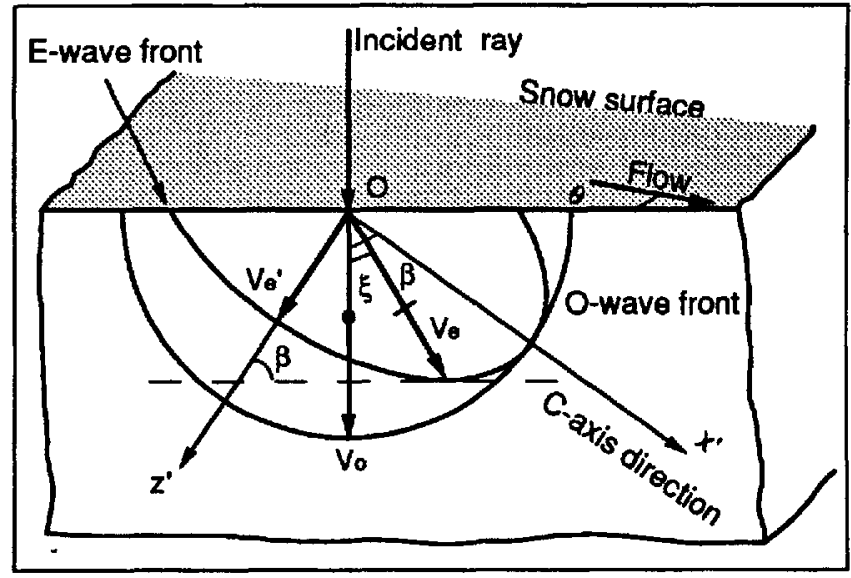

Fig. 3. Sketch of the wave fronts of the ordinary and extraordinary rays in the vertical cross-section containing the $c$ axis (principal plane). The angles $\beta$ and $\xi$ are defined in this figure, $\theta$ in Figure 2. Other quantities are explained in the text.

and ordinary waves in ice is less than 2\% (Von Hippel and others, 1969; Woodruff and Doake, 1979; Fujita and others, 1993), so $\xi<0.63^{\circ}$. For our purposes, the ordinary and extraordinary waves, therefore, can be considered as propagating in the same direction.

The phase difference between the reflected ordinary and extraordinary waves is, from Equation (3),

$$
\phi=\frac{4 \pi f h}{V_{0}}\left[\left(1+\left(\frac{n_{c}{ }^{2}}{n_{0}{ }^{2}}-1\right) \sin ^{2} \beta\right)^{\frac{1}{2}}-1\right]
$$

where $f$ is the frequency. Since $V_{\mathrm{e}}^{\prime} \approx V_{0}$, the two-way travel time of both waves is approximately $t=2 h / V_{0}$, and by Taylor expansion

$$
\phi=\pi f t\left(\frac{n_{\mathrm{c}}{ }^{2}}{n_{0}{ }^{2}}-1\right) \sin ^{2} \beta,
$$

so

$$
\beta=\sin ^{-1}\left[\frac{\phi}{\pi f t\left(n_{\mathrm{e}}^{2} / n_{\mathrm{o}}^{2}-1\right)}\right]^{\frac{1}{2}} .
$$

Note that there is more than one solution for the $c$-axis tilt, $\beta$, because our technique yields only $\cos \phi$, not $\phi$.

\section{$c$ axes distributed uniformly in a cone}

If the $c$ axes are distributed evenly in a cone, the axis of the cone becomes the effective $c$ axis, and the extraordinary-wave speed is between $V_{o}$ and $V_{\mathrm{e}}^{\prime}$. However, each of the equations for the uniaxial model is still true if one substitutes an effective refractive index for $n_{\mathrm{e}}$. That effective refractive index can be calculated for any assumed cone angle.

\section{$c$ axes distributed randomly in the principal plane}

Under some circumstances, the $c$ axes rather than clustering around a single direction tend to be distributed 

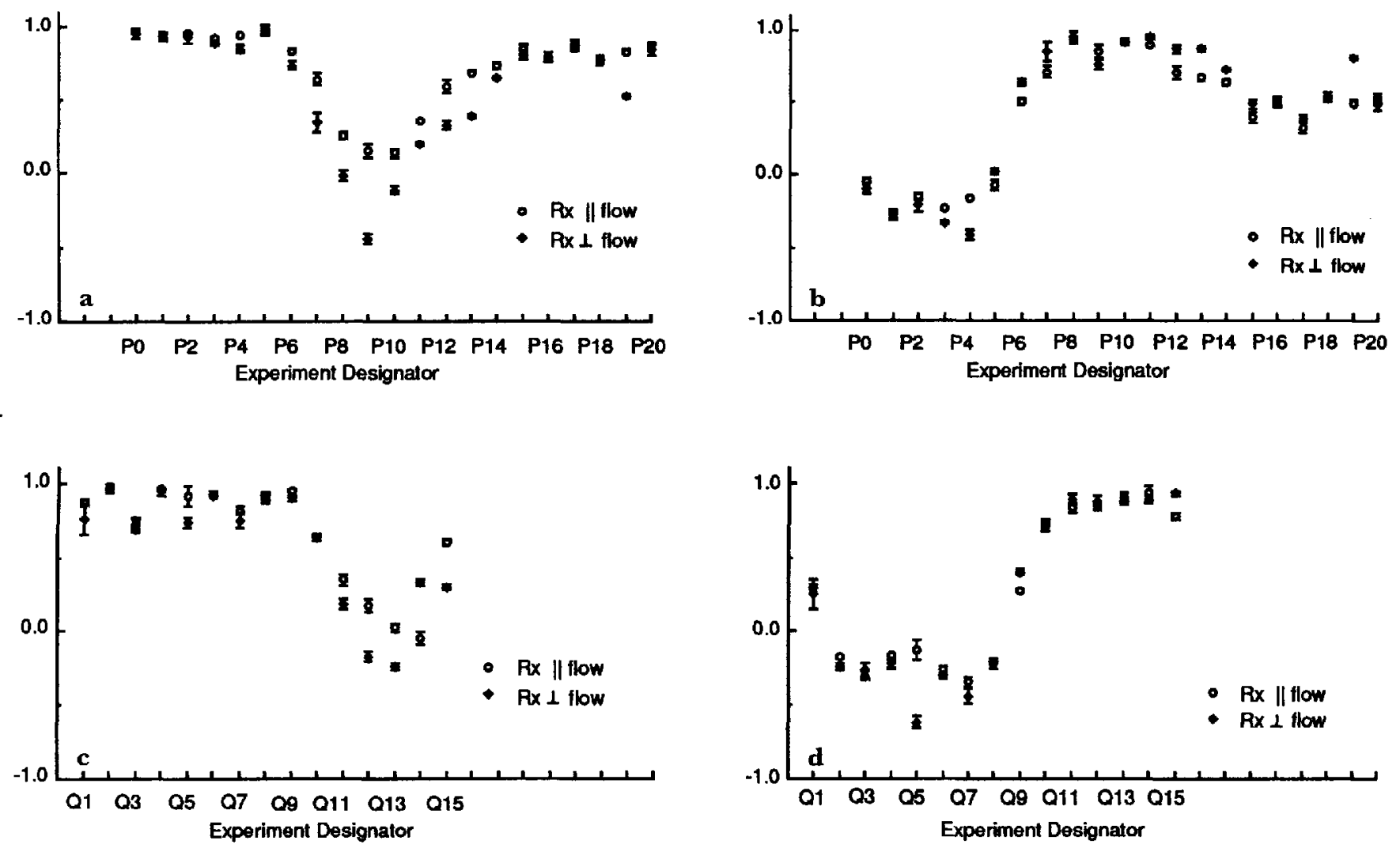

Fig. 4. Intermediate solutions $X$ and $Y$ and their standard deviations. a. $X$ values on the $P$ line; $b . Y$ values on the $P$ line; c. $X$ values on the $Q$ line; $d$. $Y$ values on the $Q$ line.

randomly in the principal plane. In this case, the average extraordinary-wave speed, $\bar{V}_{\mathrm{e}}$, is, closely enough the rangc of $V_{\mathrm{e}}$ is so small that averaging slownesses would give a negligibly different result):

$$
\bar{V}_{\mathrm{e}}=\frac{2}{\pi} \int_{0}^{\pi / 2} V_{\mathrm{c}} \mathrm{d} \beta
$$

Since $V_{0}-V_{\mathrm{e}}^{\prime} \ll V_{0}$, we can approximate the elliptical velocity function for the extraordinary wave by

$$
V_{\mathrm{c}}=V_{o}-\left(V_{o}-V_{c}^{\prime}\right) \sin ^{2} \beta
$$

integration then yields

$$
\overrightarrow{V_{\mathrm{e}}}=\frac{1}{2}\left(V_{\mathrm{o}}+V_{\mathrm{e}}^{\prime}\right) .
$$

The phase difference between the two waves is, from Equation (3),

$$
\phi=\frac{4 \pi f h}{V_{0}} \cdot \frac{n_{\mathrm{c}}-n_{\mathrm{o}}}{n_{\mathrm{o}}+n_{\mathrm{c}}}
$$

\section{RESULTS}

In our field measurements no clear and consistent internal reflections were seen, so only bottom echoes were analyzed. The bottom echoes were about $1 \mu$ s long. We assume a uniform, single layer of ice and an isotropic reflection coefficient.

Figurc 4 shows the values of $X$ and $Y$ calculated from Fquations (4) and (5). There are two scts of cach for each site, corresponding to the two orientations of the receiving intenna. The error bars depict the standard deviations of the values obtained from the 24 orientations of the transmitting antenna. Notc that the standard deviations are all small and that pairs of values are mostly in good agreement.

'The corresponding values of $\theta$ and $\cos \phi$ are shown in Figures 5-7. We plot $\cos \phi$ (Fig. 7) rather than $\phi$ to avoid multiple values, which we shall consider below. We wish to depict the different possible values of $\theta$, however, so they are shown in Figures 5 and 6.

Setting the matter of multiple values aside for the moment, we note first that the individual values of $\theta$ and $\cos \phi$ are not well determined, despite the small errors in $X$ and $Y$. That is because $\theta$ and $\cos \phi$ become independent of $X$ and $Y$ for particular valucs $\left(\cos \phi=1 ; \theta=0^{\circ}, 90^{\circ}\right.$; as those values are approached, $\theta$ and $\cos \phi$ become increasingly sensitive to small changes in $X$ and $Y$.

To see this, we consider the standard deviations of $\theta$ and $\cos \phi$ :

$$
m_{\theta}=\left(E_{X} m_{X}^{2}+E_{Y} m_{Y}^{2}\right)^{\frac{1}{2}}
$$

and

$$
m_{\cos \phi}=\left(F_{X} m_{X}^{2}+F_{Y} m_{Y}^{2}\right)^{\frac{1}{2}}
$$


a

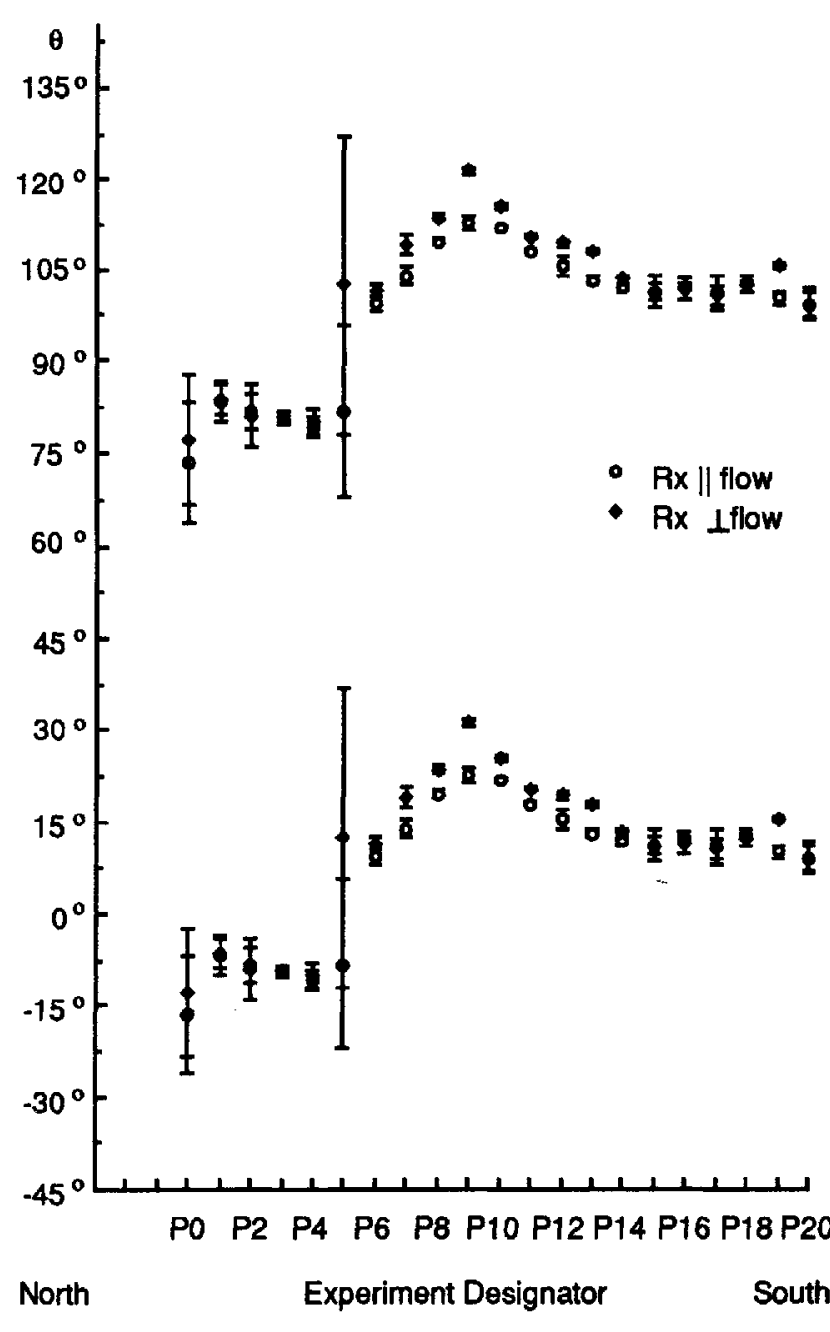

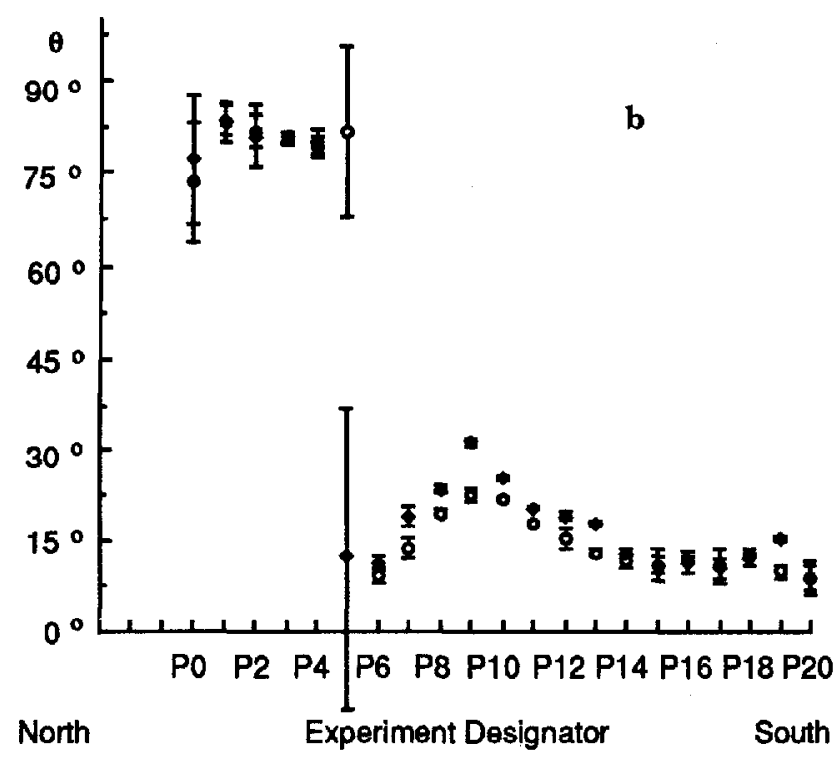

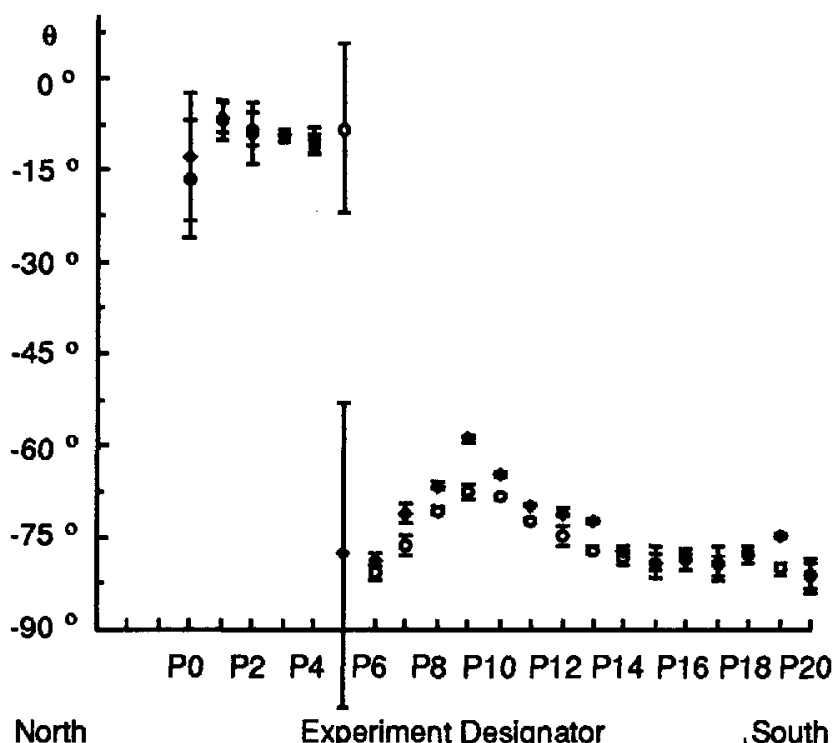

Fig. 5. Plots of $\theta$, the angle in the horizontal plane between flow and the axis of symmetry, on the $P$ line. Values for the two orienlations of the receiving antenna are shown separately. Error bars denote standard deviations. The experiments are spaced $100 \mathrm{~m}$ apart (see Fig. 1). The lefthand and righthand sets of figures each show alternative models; those in (a) are for the smaller discontinuity at P5; those in (b) are for the larger discontinuity at P5.

where $m_{X}$ and $m_{Y}$ are the standard deviations of $X$ and $Y$ and $E_{X}, E_{Y}, F_{X}$ and $F_{Y}$ are the coefficients for error propagation:

$$
E_{X}=\left[\frac{1}{2 \tan 2 \theta(1-\cos \phi)}\right]^{2}, \quad E_{Y}=\left[\frac{1}{2(1-\cos \phi)}\right]^{2}
$$

and

$$
F_{\mathrm{X}}=\left[\frac{\cos 4 \theta}{\sin ^{2} 2 \theta}\right]^{2}, \quad F_{Y}=\left[\frac{2(1+\cos 4 \theta)}{\sin 4 \theta}\right]^{2}
$$

$E_{X}, E_{Y}, F_{X}$ and $F_{Y}$ are plotted in Figure 8 as functions of $\theta$ and $\cos \phi$ (note that $E_{Y}$ is independent of $\theta$ and that $F_{X}$ and $F_{Y}$ are both independent of $\phi$ ).

From Figure 8 , we can sec why both $\theta$ and $\cos \phi$ are more poorly determined in the northern parts of the profiles in Figures $5-7$ (P0-P5, Q1-Q8) than in the southern parts - $\theta$ is scattered because $\cos \phi$ is near 1 and $\cos \phi$ is scattered because $\theta$ is near $90^{\circ}$.

Despite these factors, it is clear that there are abrupt changes in $\theta$ between P4 and P6 (Fig. 5) and between Q8 and $Q 9$ (Fig. 6); marked changes in $\cos \phi$ also appear in the, same places (Fig. 7). These step changes stem from discontinuities in $Y$ (Fig. 4). The amount of change in $\theta$ is not certain, however, because of its double valucdness. The smaller possible change in $\theta$ is about $30^{\circ}$, with values centered around either $0^{\circ}$ or $90^{\circ}$ (Figs $5 \mathrm{a}$ and $6 \mathrm{a}$ ), but a larger step of about $60^{\circ}$ cannot be ruled out (Figs $5 \mathrm{~b}$ and $6 \mathrm{~b}$ ).

To us, the large and abrupt changes argue against anisotropic reflection as the principal cause of depolarization. Not only is there no known model for an anisotropic reflection coefficicnt that would mimic such phase shifts, it is hard to see how an actively deforming bed, the anisotropic characteristics of which (if any) would have to be tied to its movement along the flow direction, could show such a rapid lateral change where 

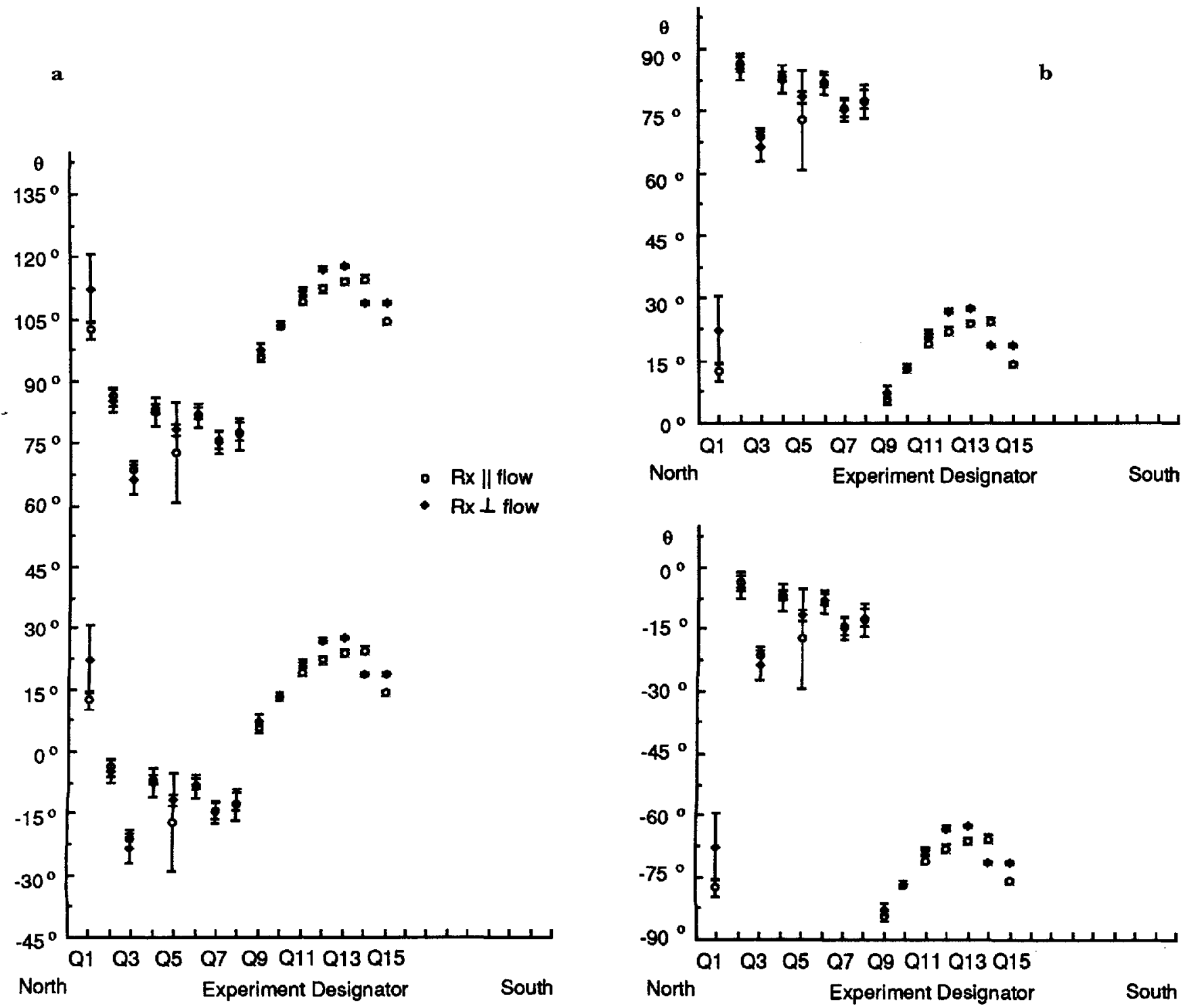

Iig. 6. Plots of $\theta$, the angle in the horizontal plane between flow and the axis of symmetry, on the Q line. Values for the lwo orienlations of the receiving antenna are shown separately. Error bars denole standard deviations. The experiments are spaced $100 \mathrm{~m}$ apart (see Fig. 1). The lefthand and righthand sets of figures each show alternative models; those in (a) are for the smaller discontinuity around $Q 9$; those in (b) are for the larger discontinuity around $Q 9$.

there is no discontinuity in flow. Anisotropic absorption could conceivably cause the observed effect but, since it has never been reported in icc, whereas anisotropy in the permittivity of an ice crystal is a known phenomenon, we will procecd on the assumption that the observed depolarization arises from the latter.

We first apply the uniaxial model. To calculate the dip of the effective $c$ axis, $\beta$ (Equation (8)), we adopt the permitlivity values of Fujita and others (1993): $\epsilon{ }^{\prime}=$ $3.189 \pm 0.006$ and $\epsilon_{1}^{\prime}=3.152 \pm 0.003 ;$ then $n_{\mathrm{c}}=\sqrt{\epsilon_{\|}^{\prime}}$ and $n_{0}=\sqrt{\epsilon_{\perp}}$. There are seven options for $\beta$, ranging from $10^{\circ}$ to $80^{\circ}$, because only $\cos \phi$ is known; $\phi$ could theoretically have a value as large as $7.5 \pi$ for horizontal $c$ axes, corresponding to $\beta=90^{\circ}$. In reality, of course, the $c$ axes are never perfectly aligned. A conical distribution of $c$ axes would yield an effective anisotropy less than the single-crystal value by an amount that would depend on the details of the distribution. The effect of that would be to reduce the maximum possible phase difference, probably by a substantial amount, and thus to eliminate the smallest values of $\phi$ (nearest-to-vertical orientations) from the allowable set. Another factor that would tend to reduce the allowable set further is that the whole ice shcct is probably not characterized by the same anisotropy; certainly, it cannot be expected to extend right to the surface (Blankenship, 1989). Reducing the fraction of the ice sheet to which the single-crystal model applies increases the required effect from that fraction.

If a conical model applies, therefore, the axis of symmetry is not near vertical. It is likely for theoretical reasons that the axis is along onc of the principal stresses (Alley, 1992) and the absence of strong shcar stress at the bed (Kamb and Engelhardt, 1991) means that the principal stress axes arc probably nearly vertical and horizontal. It follows, then, that the axis of symmetry of the ice fabric is probably nearly horizontal.

If we take as an alternative model, $c$ axes distributed randomly in the verical plane normal to flow, as found from seismic work near UpB Camp (Blankenship, 1989), the phase difference between the two waves over the 

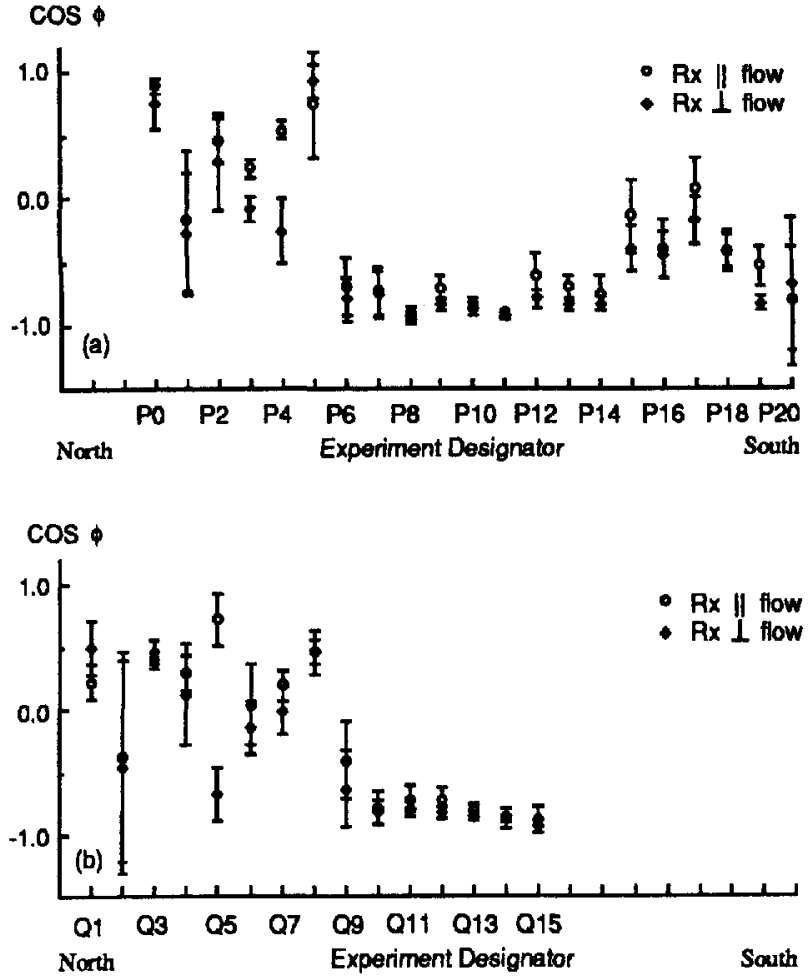

Fig. 7. Cosines of the phase differences between ordinarywiave and extraordinary-wave reflections. Values for the two orientations of the receiving antenna are shown separately. Error bars denote standard deviations. The experiments are spaced $100 \mathrm{~m}$ apart (see Fig. 1). a. Pline; b. Qline.

whole ice thickness, according to Equation (9), is $3.9 \pi$. This would also be reduced by a spreading of the $c$-axial directions out of the principal plane and by the applicability of the model to less than the entire ice column. Nevertheless, there is still ample phase difference available in the alternative model to accommodate any observed value of $\cos \phi$. 'This model also implies a horizontal axis of symmetry.

Whatever the ambiguity in the specific $c$-axis model, the results show strikingly differcnt characteristics of the icc on opposite sides of a boundary that runs parallel to flow (dotted line in Figure 1). This contrasi supports the concept of two distinct blocks of ice with different fabrics, hence different stress/strain histories.

We can use the seismic measurements Blankenship, 1989) to help choose between models. Projecting the seismic site directly upflow would put it $23 \mathrm{~km}$ to the south of profiles $\mathbf{P}$ and $\mathrm{Q}$. It is likely, then, that the $c$ axes on the southern parts of the profiles also lie in a transverse vertical plane, as they do at the seismic site. This would mean that $\theta \approx 90^{\circ}$, which would in turn argue against the validity of the lower model in Figures $5 a$ and $6 a$ and the upper model in Figures $5 \mathrm{~b}$ and $6 \mathrm{~b}$.

Another clue comes from strain measurements. Shear strains in the horizontal plane should be sensitive to the orientation of the $c$ axes in the horizontal plane, since the shear stress should change in some smooth way across the ice stream. Thus, it seems likely that a shift in $c$-axis oricntation from nearly normal to flow to nearly along
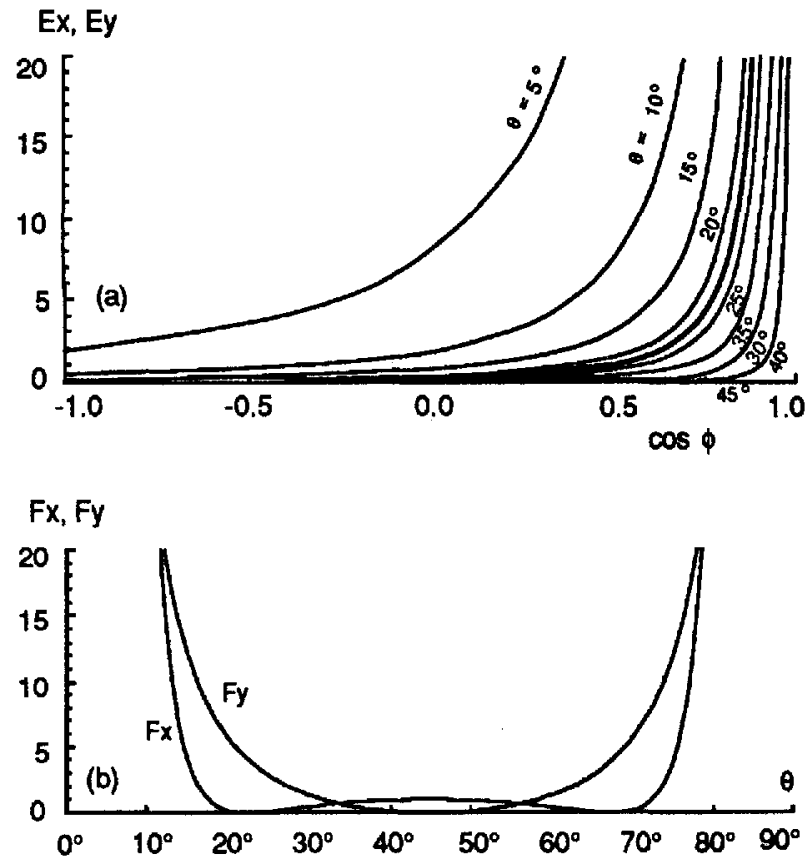

Fig. 8. a. The coefficients of error propagation for $m_{\theta}$, the standard deviation of $\theta$ values. Light lines show: $E_{X}$; heazy line showes $E_{Y}$. b. The coefficients of error propagation for $m_{\cos \phi}$, the standard deviation of $\cos \phi$ ialues.

flow across the boundary between the northern and southern zones in the profiles would produce anomalous shear strains. However, the recent survey by Hulbe and Whillans (1994) does not show any abnormal shcar strains in this location, so we conclude that the models of Figures $5 \mathrm{~b}$ and $6 \mathrm{~b}$ are less likely than those of Figures $5 \mathrm{a}$ and $6 \mathrm{a}$. We are left, then, with the upper models in Figures $5 \mathrm{a}$ and $6 \mathrm{a}$ as the ones we believe are closest 10 being correct.

\section{CONGLUSIONS}

Radar-polarization studics strongly indicate the existence, at a location on Ice Stream B, of an abrupt change in crystalline fabric across a distance of only 100 or $200 \mathrm{~m}$ perpendicular to flow. From several models that fit the data, we prefer the one that is consistent with local strain data and with fabrics estimated from seismic measurements $10 \mathrm{~km}$ downstream. In this model, the axis of symmetry of the fabric is rotated by $30^{\circ}$ across the boundary, from $+15^{\circ}$ to $15^{\circ}$ relative the direction transverse to flow, as if the two parts of the profile lay on blocks of ice with different stress/strain histories. It is even possible that profile $Q$ spans a block - note that the orientation at the most northcrly site, Ql, is "normal", i.e. the same as for the southern (righthand) section. 'The occurrence of exotic blocks within ice steams has been suggested by several authors in relation to ice rafts (Bindschadler and others, 1987), discontinuity in mass flux (Shabtaie and others, 1988) and irregular features within the ice stream (Whillans, 1987). 


\section{ACKNOWLEDGEMENTS}

The authors would like to thank T.S. Clarke and A. N. Novick for their help with the field work. This paper was supported by U.S. National Science Foundation grant DPP-90-18530. This is contribution No. 543 of the Geophysical and Polar Research Center, Lniversity of Wisconsin Madison.

\section{REFERENCES}

Alley, R. B. 1988. Fabrics in polar ice sheets: development and prediction. Science, 240 4851$)$, 493-495.

Alley, R.B. 1992. Flow-law hypotheses for icc-sheet modeling. J. - Glaciol., 38(129), 245-256.

Bentlcy, C. R. 1975. Advances in geophysical exploration of ice sheets and glaciers. J. Glaciol., 15(73), 113-135.

Bindschadler, R. A., S. N. Stephenson, D. R. MacAyeal and S. Shabtaie. 1987. Ice dynamics at the mouth of Ice Stream B, Antarctica. $f$. Geophys. Res., 92(B9), 8885-8894.

Blankenship. D.D. 1989. Seismological investigations of a West Antarctic ice stream. (Ph.D. thesis, University of WisconsinMadison.)

Cliarke, I.S. and C.R. Bentley. 1994. High-resolution radar on Ice Stream B2, Antarctica: measurements of electromagnetic wave specd in firn and strain history from buried crevasses. Ann. Glaciol., $\mathbf{2 0}$ (see paper in this volume).

Doake, C.S. M. 1981. Polarization of radio waves in icc shects. Ceophys. 7. R. Astron. Soc., 64, 539-558.

Fujita, S., S. Mac and T. Matsuoka. 1993. Dielectric anisotropy in ice Ih at 9.7 ( $\mathrm{H}_{z .}$ Ann. Glaciol, 17, 276-280).
Hargreaves, N.D. 1977. The polarization of radio signals in the radio echo sounding of ice sheets. 7. Phys. D, 10, 12851304.

Hargreaves. N. D. 1978. The radio frequency birefringence of polar ice. 7. Glaciol, 2185 ), 301-313

Hippel, A. von, D. B. Knoll, W. B. Westphal, M. A. Maidique, J. Iglesias and R. Mykolajewycz. 1969. The dielectric relaxation spectra of water, ice and aqueous solutions and their interpret-ation. VI. New measurements on relaxation and conduction of "pure" ice Ih single crystals and their critical evaluation. Cambridge, MA, Massachusetts Institute of Technology, Iaboratory for Insulation Research. (Technical Report 6.)

Hulbe, C. L. and I. M. Whillans. 1994. Evaluation of strain rates on Ice Strcam B, Antarctica, obtained using GPS phase measurements. Ann. Glaciol., 20 (sce paper in this volume).

Jiracek, G.R. 1967. Radio sounding of Antarctic ice. Eniversity of Wisconsin-Madison Geophysical and Polar Research Center. Res. Rep. Ser. 67-1.

Kamb, B. and H. Fngclhardt. 1991. Antarctic Ice Stream B: conditions controlling its motion and interactions with the climate system. International Association of IIydrologiral Sciences Pubitation 208 (Symposium at St. Petersburg 1990 - Glacien-Ocean-Atmosphere Interactions), $145-154$.

Shabtaie, S., C. R. Bentley, R. A. Bindschadler and D. R. MacAyeal. 1988. Mass-balance studics of Icc Streams A, B, and C, West Antarctica, and possible surging behavior of Ice Stream B. Ann. Glaciol., 11, 137-149.

Whillans, I. M. 1987. Flow of Ice Streams B and C. Ant. J. L.S., 22(5), $67-68$.

Woodruff, A. H. W. and C.S. M. Doake. 1979. Depolarization of radio waves can distinguish between floating and grounded ice sheets. $J$ Glaciol., $23(89), 223232$.

The accuracy of references in the text and in this list is the responsibility of the authors, to whom queries should be addressed. 